Annales Academiæ Scientiarum Fennicæ

Mathematica

Volumen 38, 2013, 535-546

\title{
FAT AND THIN SETS FOR DOUBLING MEASURES IN EUCLIDEAN SPACE
}

\section{Wen Wang, Shengyou Wen* and Zhi-Ying Wen}

\author{
Yunnan University, Department of Mathematics \\ Kunming 650091, P. R. China; sophia_84@126.com \\ Hubei University, Department of Mathematics \\ Wuhan 430062, P. R. China; sywen_65@163.com \\ Tsinghua University, Department of Mathematics \\ Beijing 100084, P. R. China; wenzy@tsinghua.edu.cn
}

\begin{abstract}
According to the size of sets for doubling measures, subsets of $\mathbf{R}^{n}$ can be divided into six classes. Sets in these six classes are respectively called very thin, fairly thin, minimally thin, minimally fat, fairly fat, and very fat. In our main results, we prove that if a quasisymmetric mapping $f$ of $[0,1]$ maps a uniform Cantor set $E$ onto a uniform Cantor set $f(E)$, then $E$ is of positive Lebesgue measure if and only if $f(E)$ is so. Also, we prove that the product of $n$ uniform Cantor sets is very fat if and only if each of the factors is very fat, and that the product is minimally fat if and only if one of the factors is minimally fat.
\end{abstract}

\section{Introduction}

A Borel regular measure $\mu$ on $\mathbf{R}^{n}$ is called doubling if there is a constant $K>1$ such that

$$
0<\mu\left(Q_{1}\right) \leq K \mu\left(Q_{2}\right)<\infty
$$

for any pair $Q_{1}, Q_{2}$ of adjacent cubes of the same side-length in $\mathbf{R}^{n}$. In this case, we also say that $\mu$ is $K$-doubling. Hereafter, a cube $Q(x, r)$ in $\mathbf{R}^{n}$, of side-length $2 r$ centered at $x=\left(x_{1}, \cdots, x_{n}\right)$, is defined to be the product set

$$
\prod_{i=1}^{n}\left[x_{i}-r, x_{i}+r\right] \text {. }
$$

Two cubes are said to be adjacent, if they have a nonempty intersection.

Denote by $\mathcal{D}\left(\mathbf{R}^{n}\right)$ the family of doubling measures on $\mathbf{R}^{n}$ and by $\mathcal{D}_{K}\left(\mathbf{R}^{n}\right)$ the family of $K$-doubling measures. Then $\mathcal{D}_{K}\left(\mathbf{R}^{n}\right)$ is increasing with respect to $K$ and $\mathcal{D}\left(\mathbf{R}^{n}\right)=\cup_{K>1} \mathcal{D}_{K}\left(\mathbf{R}^{n}\right)$. For every set $E \subset \mathbf{R}^{n}$ denote by $|E|$ its $n$-dimensional Lebesgue measure and by $\operatorname{dim}_{H} E$ its Hausdorff dimension.

We divide subsets of $\mathbf{R}^{n}$ into six classes: VF, FF, MF, VT, FT, and MT. Sets in these six classes are respectively called very fat, fairly fat, minimally fat, very thin, fairly thin, and minimally thin. They are defined as follows: Let $E \subset \mathbf{R}^{n}$.

$E \in \mathrm{VF}$ if $\mu(E)>0$ for all $\mu \in \mathcal{D}\left(\mathbf{R}^{n}\right)$.

$E \in \mathrm{MF}$ if $|E|>0$ and for every $K>1$ there is a $K$-doubling measure $\mu \in$ $\mathcal{D}_{K}\left(\mathbf{R}^{n}\right)$ such that $\mu(E)=0$.

doi:10.5186/aasfm.2013.3827

2010 Mathematics Subject Classification: Primary 28A12; Secondary 30L10.

Key words: Uniform Cantor set, doubling measure, quasisymmetric mapping, fat set, thin set. Supported by NSFC (No. 11271114 and No. 10971056) and by Morningside Center of Mathematics, Chinese Academy of Sciences.

${ }^{*}$ Corresponding author. 
$E \in \mathrm{FF}$ if $|E|>0$ and $E \notin \mathrm{VF} \cup \mathrm{MF}$.

$E \in \mathrm{VT}$ if $\mu(E)=0$ for all $\mu \in \mathcal{D}\left(\mathbf{R}^{n}\right)$.

$E \in \mathrm{MT}$ if $|E|=0$ and for every $K>1$ there is a $K$-doubling measure $\mu \in$ $\mathcal{D}_{K}\left(\mathbf{R}^{n}\right)$ such that $\mu(E)>0$.

$E \in \mathrm{FT}$ if $|E|=0$ and $E \notin \mathrm{VT} \cup \mathrm{MT}$.

The above classification of subsets of $\mathbf{R}^{n}$ was introduced by Buckley, Hanson, and MacManus [1] in 2001. To gain some understanding on fat and thin sets, let us recall some known results on them. We begin with two important facts on doubling measures.

Lemma 1. Let $K \geq 1$ and let $\mu \in \mathcal{D}_{K}\left(\mathbf{R}^{n}\right)$. Then

$$
C^{-1}\left(\frac{r}{R}\right)^{\beta} \leq \frac{\mu(Q(y, r))}{\mu(Q(x, R))} \leq C\left(\frac{r}{R}\right)^{\alpha}
$$

for any cubes $Q(y, r)$ and $Q(x, R)$ in $\mathbf{R}^{n}$ with $Q(y, r) \subset Q(x, R)$, where $C$, $\alpha$, and $\beta$ are constants depending only on $K$ and $n$.

Lemma 2. For every $\varepsilon>0$ there is a subset $E$ of $\mathbf{R}^{n}$ and a measure $\mu \in \mathcal{D}\left(\mathbf{R}^{n}\right)$ such that $\operatorname{dim}_{H} E<\varepsilon$ and $\mu(E)=\mu\left(\mathbf{R}^{n}\right)$.

Doubling measures may be defined on a metric space with cubes replaced by balls (see [10, 17]). Lemma 1 holds for doubling measures on every uniformly perfect space [7]. Lemma 2 was proved by Wu for every compact doubling metric space [20]. It is also true for every complete doubling metric space [9]. Recently, Käenmäki, Rajala, and Suomala [11] showed that for every complete doubling metric space and for every $\varepsilon>0$ there is a doubling measure having full measure on a set of packing dimension at most $\varepsilon$. We note that for every $\varepsilon>0$ there is a unidimensional doubling measure on $\mathbf{R}^{n}$ of dimension $\varepsilon$. Recall that a Radon measure $\mu$ on $\mathbf{R}^{n}$ is unidimensional of dimension $\varepsilon$, if its local dimension is equal to $\varepsilon$ for $\mu$-a.e. $x \in \mathbf{R}^{n}$ (See [3]).

It is clear that a set with nonempty interior is very fat. However, a set of full Lebesgue measure may not be very fat. For the set $E$ in Lemma 2, we have that $\mathbf{R}^{n} \backslash E$ is of full Lebesgue measure, but it is not very fat.

It is easily seen from Lemma 1 that every set of $\operatorname{dim}_{H}=0$ is very thin. This can not be improved because Lemma 2 implies that for every $\varepsilon>0$ there is a set of Hausdorff dimension $\varepsilon$, which is not very thin. See also [15].

Another example of very thin sets can be obtained by porosity. A set $E \subset \mathbf{R}^{n}$ is said to be porous, if there is a constant $c>0$ such that every cube $Q(x, r)$ has a subcube of sidelength $\mathrm{cr}$ not meeting $E$. It is not difficult to see that porous sets are very thin. In fact, sets with a certain weaker porosity are very thin (see [19]). By contrast, Staples and Ward [14] introduced the $\alpha_{k}$-thickness condition for subsets of $\mathbf{R}$ and proved that if $\left\{\alpha_{k}\right\} \in \ell^{p}$ for all $0<p<1$, then $\alpha_{k}$-thick sets are very fat in $\mathbf{R}$. Hereafter $\ell^{p}$ denotes the family of sequences $\left\{a_{k}\right\}_{k=1}^{\infty}$ of positive real numbers with $\sum a_{k}^{p}<\infty$.

The above results on porosity and thickness have been extended to uniform perfect metric space by Ojala, Rajala, and Suomala [12], in which very fat and very thin sets are simply called fat and thin, respectively. They proved that the cut-out set

$$
X \backslash \bigcup_{i=1}^{\infty} B\left(x_{i}, r_{i}\right)
$$


is fat or thin, provided that $X$ is a uniform perfect metric space and $B\left(x_{i}, r_{i}\right)$ is a sequence of closed balls in $X$ with $\left\{r_{i}\right\} \in \ell^{p}$ for any $0<p<1$.

As an application of the fact that porous sets are very thin, we easily see that if $f: \mathbf{R}^{n} \rightarrow \mathbf{R}$ is Lipschitz, then the graph

$$
G(f)=\left\{(x, f(x)): x \in \mathbf{R}^{n}\right\}
$$

is very thin for doubling measures on $\mathbf{R}^{n+1}$. By contrast, Garnett, Killip, and Schul [4] constructed a rectifiable curve that is not very thin for doubling measures on $\mathbf{R}^{n}$. However, the following question is still open.

Problem 1. Is the graph of a continuous function $f:[0,1] \rightarrow[0,1]$ very thin for doubling measures on $\mathbf{R}^{2}$ ?

This paper is devoted to studying uniform Cantor sets and doubling measures. Let $\left\{n_{k}\right\}$ be a sequence of positive integers and $\left\{c_{k}\right\}$ a sequence of real numbers in $(0,1)$, with $n_{k} c_{k}<1$ for every $k \geq 1$. The uniform Cantor set of data $\left\{n_{k}\right\}$ and $\left\{c_{k}\right\}$, denoted by $E\left\{n_{k}, c_{k}\right\}$, is defined by

$$
E\left\{n_{k}, c_{k}\right\}=\bigcap_{k=0}^{\infty} E_{k},
$$

where $\left\{E_{k}\right\}$ is a nested sequence of compact sets, $E_{0}=[0,1]$, and $E_{k}$ is defined by removing $n_{k}$ open intervals of the same length $c_{k}|I|$ from every component interval $I$ of $E_{k-1}$, such that the rest $n_{k}+1$ closed intervals in $I$ are of equal length. It is clear from the definition that the set $E_{k}$ consists of

$$
N_{k}=\prod_{i=1}^{k}\left(n_{i}+1\right)
$$

closed intervals, each of length

$$
\delta_{k}=\prod_{i=1}^{k} \frac{1-n_{i} c_{i}}{n_{i}+1} .
$$

The Lebesgue measure of $E\left\{n_{k}, c_{k}\right\}$ is

$$
\left|E\left\{n_{k}, c_{k}\right\}\right|=\prod_{i=1}^{\infty}\left(1-n_{i} c_{i}\right),
$$

and so

$$
\left|E\left\{n_{k}, c_{k}\right\}\right|>0 \Longleftrightarrow\left\{n_{i} c_{i}\right\} \in \ell^{1} .
$$

Lemma 3. Let $E=E\left\{n_{k}, c_{k}\right\}$ be a uniform Cantor set and $0<p<\infty$. Then there is a $K_{p}$-doubling measure $\mu$ on $[0,1]$ such that

$$
\left(1-C_{p}\left(n_{k} c_{k}\right)^{p}\right) \mu\left(E_{k-1}\right) \leq \mu\left(E_{k}\right) \leq\left(1-C_{p}^{-1}\left(n_{k} c_{k}\right)^{p}\right) \mu\left(E_{k-1}\right),
$$

where $K_{p}$ and $C_{p}$ are constants depending only on $p$. Moreover, $K_{p}$ can be chosen such that $\lim _{p \rightarrow 1} K_{p}=1$.

For the proof of this lemma we refer to [13]. See also Csörnyei and Suomala [2]. It plays an important role in proving the following result (see $[1,6,13]$ ).

Lemma 4. Let $E=E\left\{n_{k}, c_{k}\right\}$ be a uniform Cantor set. Then

(a) $E \in \mathrm{VF}$ if and only if $\left\{n_{k} c_{k}\right\} \in \cap_{p>0} \ell^{p}$; 
(b) $E \in M F$ if and only if $\left\{n_{k} c_{k}\right\} \in \ell^{1} \backslash \cup_{p<1} \ell^{p}$;

(c) $E \in \mathrm{VT}$ if and only if $\left\{n_{k} c_{k}\right\} \notin \cup_{p>1} \ell^{p}$;

(d) $E \in \mathrm{MT}$ if and only if $\left\{n_{k} c_{k}\right\} \in \cap_{p>1} \ell^{p} \backslash \ell^{1}$.

A sufficient and necessary condition for fairly fat (fairly thin, respectively) uniform Cantor sets is implicated in the lemma itself. Using Lemma 4, we may construct a very thin set $E \subset \mathbf{R}$ of $\operatorname{dim}_{H} E=1$.

We have seen that the size of sets in the sense of doubling measures can not be described by Hausdorff dimension. In the case of $n=1$, we have a satisfactory criterion for very fat and very thin sets (see [18]). In the present paper, we continue to investigate fat and thin sets in $\mathbf{R}^{n}$. We shall focus on the following two questions:

1. For which doubling measures $\mu$ on $[0,1]$ we have $\mu(E)>0$, provided that the uniform Cantor set $E$ in Lemma 4 is neither VF nor VT?

2. When does the product of $n$ subsets of $[0,1]$ belong to VF, FF, MF, VT, FT, or MT for doubling measures on $[0,1]^{n}$ ?

To formulate our result for Question 1, we recall that a homeomorphism $f: \mathbf{R}^{n} \rightarrow$ $\mathbf{R}^{n}$ is quasisymmetric, if there is a constant $K$ such that

$$
|f(x)-f(a)| \leq K|f(x)-f(b)|
$$

for every triple $a, b, x$ of distinct points in $\mathbf{R}^{n}$ with $|x-a| \leq|x-b|$ (see [7]). Then it is clear that, for $n=1$, a measure $\mu$ on $\mathbf{R}$ is doubling if and only if $\mu$ is the pullback measure $m \circ f$ of a quasisymmetric mapping $f: \mathbf{R} \rightarrow \mathbf{R}$. Our first result, formulated in Theorem 1, partly answers Question 1.

Theorem 1. Let $E$ be the uniform Cantor set of data $\left\{n_{k}\right\}$ and $\left\{c_{k}\right\}$. Let $f:[0,1] \rightarrow[0,1]$ be a quasisymmetric mapping such that $f(E)$ is the uniform Cantor set of data $\left\{n_{k}\right\}$ and $\left\{d_{k}\right\}$. Suppose that every component interval of level- $k$ of $E$ is mapped onto a component interval of level- $k$ of $f(E)$. Then $|E|>0$ if and only if $|f(E)|>0$.

Let $E$ be a uniform Cantor set and $f:[0,1] \rightarrow[0,1]$ a quasisymmetric mapping. We say that $f(E)$ has uniformly comparable gaps, if there is a constant $K>1$ such that

$$
|f(J)| \leq K|f(M)|
$$

for every $k \geq 1$ and for every pair $J, M$ of component intervals of $E_{k-1} \backslash E_{k}$.

Corollary 1. Let $E$ be a uniform Cantor set. Let $f:[0,1] \rightarrow[0,1]$ be a quasisymmetric mapping such that $f(E)$ has uniformly comparable gaps. Then $|E|>0$ implies $|f(E)|>0$.

It is known that for the uniform Cantor set $E$ of data $\left\{n_{k}\right\}$ and $\left\{c_{k}\right\}$, we have $\operatorname{dim}_{H} f(E)=1$ for any quasisymmetric mapping $f$ of $[0,1]$ onto itself, if $\left\{n_{k}\right\}$ is bounded and $\operatorname{dim}_{H} E=1$ (see [5, 8]). By contrast, Corollary 1 gives us a condition under which quasisymmetric mappings map a uniform Cantor sets of positive length onto a set of positive length. It should be pointed out that Corollary 1 had been obtained for $\alpha_{k}$-thick sets by Staples and Ward [14] in a different way.

We do not know if Theorem 1 is true without assuming that every component interval of level- $k$ of $E$ is mapped onto a component interval of level- $k$ of $f(E)$. Also, the following question is open. 
Problem 2. Does $|E|=0$ imply $|f(E)|=0$ under the assumption of Corollary 1 ?

When $n \geq 2$ we have no problem of Theorem 1 for quasisymmetric mappings because, in this case, the quasisymmetry is equivalent to the quasiconformality and the pullback measures $m \circ f$ by quasisymmetric mappings are mutually absolutely continuous with the Lebesgue measure [16]. However, the question of which sets are fat or thin for doubling measures is still pending. Now we are going to state our results in this direction.

Theorem 2. Let $E_{1}, \cdots, E_{n}$ be sets in $[0,1]$ of Lebesgue measure zero. Then

(1) $E_{1} \times \cdots \times E_{n} \in \mathrm{VT}$ if and only if $E_{i} \in \mathrm{VT}$ for some $i$,

(2) $E_{1} \times \cdots \times E_{n} \in \mathrm{MT}$ if and only if $E_{i} \in \mathrm{MT}$ for all $i$.

Theorem 3. Let $E_{1}, \cdots, E_{n}$ be sets in $[0,1]$ of positive Lebesgue measure. Then

(1) if $E_{1} \times \cdots \times E_{n} \in \mathrm{VF}$ then $E_{i} \in \mathrm{VF}$ for all $i$,

(2) if $E_{i} \in \mathrm{MF}$ for some $i$ then $E_{1} \times \cdots \times E_{n} \in \mathrm{MF}$.

We do not know if the inverses of two propositions in Theorem 3 hold for the product of general sets. We shall prove that they are true for products of uniform Cantor sets.

Theorem 4. Let $E_{1}, \cdots, E_{n}$ be uniform Cantor sets in $[0,1]$ of positive Lebesgue measure. Then

(1) $E_{1} \times \cdots \times E_{n} \in \mathrm{VF}$ if and only if $E_{i} \in \mathrm{VF}$ for all $i$,

(2) $E_{1} \times \cdots \times E_{n} \in \mathrm{MF}$ if and only if $E_{i} \in \mathrm{MF}$ for some $i$.

Theorems 2, 3, and 4 will be proved only for $n=2$ because, in higher dimensional case, the related things are the same. The uniformity assumption for Cantor sets in Theorems 1 and 4 is unnecessarily too strong. It would be interesting to obtain further results for more general Cantor sets.

\section{Proof of Theorem 1}

Let $E$ be a uniform Cantor set of data $\left\{n_{k}\right\}$ and $\left\{c_{k}\right\}$. Let $f:[0,1] \rightarrow[0,1]$ be a quasisymmetric mapping such that $f(E)$ is a uniform Cantor set of data $\left\{n_{k}\right\}$ and $\left\{d_{k}\right\}$. Suppose that every component interval of level- $k$ of $E$ is mapped onto a component interval of level- $k$ of $f(E)$. We are going to prove that $|E|>0$ if and only if $|f(E)|>0$. Since the inverse of a quasisymmetric mapping is quasisymmetric, it suffices to show that $|E|>0$ implies $|f(E)|>0$.

Let $\mathcal{G}_{k}$ denote the family of component intervals of $E_{k-1} \backslash E_{k}$. A member in $\mathcal{G}_{k}$ will be called a gap of level $k$ of $E$. Write $I \in E_{k}$ when $I$ is a component interval of $E_{k}$. The proof is based on the following lemmas.

Lemma 5. Let $s \geq k$ be positive integers. Let $I_{k}$ be a component interval of $E_{k}$ and $I_{s}$ a component interval of $E_{s}$. Then

$$
\frac{\left|f\left(I_{s}\right)\right|}{\left|f\left(I_{k}\right)\right|} \leq|E|^{-1} \frac{\left|I_{s}\right|}{\left|I_{k}\right|}
$$

Proof. By the assumptions, we have

$$
\frac{\left|f\left(I_{s}\right)\right|}{\left|f\left(I_{k}\right)\right|}=\prod_{i=k+1}^{s} \frac{1-n_{i} d_{i}}{n_{i}+1} \leq\left(\prod_{i=k+1}^{s}\left(1-n_{i} c_{i}\right)\right)^{-1} \prod_{i=k+1}^{s} \frac{1-n_{i} c_{i}}{n_{i}+1} \leq|E|^{-1} \frac{\left|I_{s}\right|}{\left|I_{k}\right|} .
$$


This proves the lemma.

Lemma 6. Let $s \geq k$. Let $I_{s} \in E_{s}, I_{k} \in E_{k}, I_{s+1} \in E_{s+1}$, and $J_{s+1} \in \mathcal{G}_{s+1}$ with $I_{s+1} \cup J_{s+1} \subset I_{s} \subset I_{k}$. Then

$$
\frac{\left|f\left(I_{s+1} \cup J_{s+1}\right)\right|}{\left|f\left(I_{k}\right)\right|} \leq \frac{2|E|^{-1}}{\left(n_{s+1}+1\right)} \frac{\left|I_{s}\right|}{\left|I_{k}\right|} .
$$

Proof. By the construction of the uniform Cantor set $E$ and the proof of Lemma 5 , we have

$$
\begin{aligned}
\frac{\left|f\left(I_{s+1} \cup J_{s+1}\right)\right|}{\left|f\left(I_{k}\right)\right|} & =\frac{\left|f\left(I_{s+1}\right)\right|}{\left|f\left(I_{k}\right)\right|}+\frac{\left|f\left(J_{s+1}\right)\right|}{\left|f\left(I_{k}\right)\right|}=\prod_{i=k+1}^{s+1} \frac{1-n_{i} d_{i}}{n_{i}+1}+d_{s+1} \prod_{i=k+1}^{s} \frac{1-n_{i} d_{i}}{n_{i}+1} \\
& =\left(\frac{1-n_{s+1} d_{s+1}}{n_{s+1}+1}+d_{s+1}\right) \prod_{i=k+1}^{s} \frac{1-n_{i} d_{i}}{n_{i}+1} \\
& \leq \frac{1+d_{s+1}}{n_{s+1}+1}|E|^{-1} \frac{\left|I_{s}\right|}{\left|I_{k}\right|} \leq \frac{2|E|^{-1}}{\left(n_{s+1}+1\right)} \frac{\left|I_{s}\right|}{\left|I_{k}\right|}
\end{aligned}
$$

The proof of this lemma is complete.

Since $|E|>0$, we have $\left\{n_{k} c_{k}\right\} \in \ell^{1}$, so, without loss of generality, we may assume that $n_{k} c_{k}<1 / 3$ for all $k \geq 1$. Since $f(E)$ has been assumed to be the uniform Cantor set of data $\left\{n_{k}\right\}$ and $\left\{d_{k}\right\}$, by (2), to show $|f(E)|>0$, we only need to prove

$$
n_{k} d_{k} \leq K n_{k} c_{k}
$$

for all $k \geq 1$. Hereafter $K$ is a constant depending only on the quasisymmetry constant of $f$ and it may be different in every appearance.

Now, let $k$ be a given positive integer. Let $I_{k} \in E_{k}$ and $J_{k} \in \mathcal{G}_{k}$. Then

$$
n_{k} d_{k} \leq \frac{\left|f\left(J_{k}\right)\right|}{\left|f\left(I_{k}\right)\right|}
$$

On the other hand, since $n_{k} c_{k}<1 / 3$ has been assumed, one has

$$
\frac{\left|J_{k}\right|}{\left|I_{k}\right|}=\frac{\left(n_{k}+1\right) c_{k}}{1-n_{k} c_{k}} \leq 3 n_{k} c_{k}<1
$$

Let $s \geq k$ be the unique integer such that $\left|I_{s+1} \cup J_{s+1}\right| \leq\left|J_{k}\right|<\left|I_{s} \cup J_{s}\right|$, where $I_{s} \in E_{s}, J_{s} \in \mathcal{G}_{s}, I_{s+1} \in E_{s+1}$, and $J_{s+1} \in \mathcal{G}_{s+1}$. Two possible cases may happen.

Case 1. $\left(n_{s+1}+1\right)\left|I_{s+1} \cup J_{s+1}\right| \leq\left|J_{k}\right|<\left|I_{s} \cup J_{s}\right|$.

In this case, we have $\left|I_{s}\right|<\left|J_{k}\right|<2\left|I_{s}\right|$. We may assume that $I_{s}$ and $J_{k}$ are adjacent. By (6), the quasisymmetry of $f$, Lemma 5 , and (7), we get

$$
n_{k} d_{k} \leq \frac{\left|f\left(J_{k}\right)\right|}{\left|f\left(I_{k}\right)\right|} \leq K \frac{\left|f\left(I_{s}\right)\right|}{\left|f\left(I_{k}\right)\right|} \leq K|E|^{-1} \frac{\left|I_{s}\right|}{\left|I_{k}\right|} \leq K|E|^{-1} \frac{\left|J_{k}\right|}{\left|I_{k}\right|} \leq K|E|^{-1} n_{k} c_{k} .
$$

Case 2. $p\left|I_{s+1} \cup J_{s+1}\right| \leq\left|J_{k}\right|<(p+1)\left|I_{s+1} \cup J_{s+1}\right|$ for some $1 \leq p \leq n_{s+1}$. 
In this case, by (6), the quasisymmetry of $f$, Lemma 6 , and (7), we get

$$
\begin{aligned}
n_{k} d_{k} & \leq \frac{\left|f\left(J_{k}\right)\right|}{\left|f\left(I_{k}\right)\right|} \leq K \frac{p\left|f\left(I_{s+1} \cup J_{s+1}\right)\right|}{\left|f\left(I_{k}\right)\right|} \leq K \frac{\left|J_{k}\right|}{\left|I_{s+1} \cup J_{s+1}\right|} \frac{\left|f\left(I_{s+1} \cup J_{s+1}\right)\right|}{\left|f\left(I_{k}\right)\right|} \\
& \leq K \frac{\left|J_{k}\right|}{\left|I_{s+1} \cup J_{s+1}\right|} \frac{2|E|^{-1}}{\left(n_{s+1}+1\right)} \frac{\left|I_{s}\right|}{\left|I_{k}\right|} \leq 2 K|E|^{-1} \frac{\left|J_{k}\right|}{\left|I_{k}\right|} \leq 2 K|E|^{-1} n_{k} c_{k} .
\end{aligned}
$$

This proves (5). The proof of Theorem 1 is thus completed.

Proof of Corollary 1. Let $E$ be a uniform Cantor set and $f:[0,1] \rightarrow[0,1]$ be a quasisymmetric mapping such that $f(E)$ has uniformly comparable gaps. We are going to show that $|E|>0$ implies $|f(E)|>0$.

In fact, since $f(E)$ has uniformly comparable gaps, there is a constant $K>1$ such that

$$
K^{-1}|f(L \backslash E)| \leq|f(I \backslash E)| \leq K|f(L \backslash E)|
$$

for all $k$ and for all pairs $I, L \in E_{k}$. Given a $k$ and a pair $I, L \in E_{k}$, if $|f(E)|=0$, then

$$
|f(E \cap I)|=|f(E \cap L)|=0,
$$

and so $K^{-1}|f(L)| \leq|f(I)| \leq K|f(L)|$. This implies that $f(E)$ also has uniformly comparable component intervals, so there is a bilipschitz mapping $g:[0,1] \rightarrow[0,1]$ such that $g(f(E))$ is a uniform Cantor set. Noting that $g \circ f$ is quasisymmetric with $|g(f(E))|=0$, we get $|E|=0$ from Theorem 1, a contradiction.

\section{Products, projections and extensions}

To prove the rest results of this paper, we need the following facts on products, projections and extensions of doubling measures.

Lemma 7. Let $K_{i} \geq 1$ and $\mu_{i} \in \mathcal{D}_{K_{i}}([0,1]), i=1,2$. Then the product $\mu_{1} \times \mu_{2}$ is a $K_{1} K_{2}$-doubling measure on $[0,1]^{2}$.

Proof. It is immediate from the definition of doubling measure.

Lemma 8. Let $K \geq 1$ and $\mu \in \mathcal{D}_{K}\left([0,1]^{2}\right)$. Let $\delta>0$. Let $I$ and $J$ be intervals in $[0,1]$ with $|I| \leq \delta|J|$. Then the measure $\mu(\cdot \times J)$, defined on $I$, is $C(K, \delta)$-doubling on $I$, where $C(K, \delta)$ is a constant depending only on $K$ and $\delta$. Moreover, $C(K, \delta)$ can be chosen so that $\lim _{\delta \rightarrow 0} C(K, \delta)=K$.

Proof. It is not difficult to prove that the measure $\mu(\cdot \times J)$ is $C(K, \delta)$-doubling on $I$, with the constant $C(K, \delta)$ depending only on $K$ and $\delta$. We only show that $C(K, \delta)$ can be chosen so that $\lim _{\delta \rightarrow 0} C(K, \delta)=K$.

Let $\delta<1 / 2$. Let $I_{1}, I_{2}$ be adjacent intervals of equal length in $I$. Let $J=\cup_{i=1}^{k} J_{i}$ be a partition of $J$ by intervals with

$$
\left|J_{1}\right|=\left|J_{2}\right|=\cdots=\left|J_{k-1}\right|=\left|I_{1}\right|, 0 \leq\left|J_{k}\right|<\left|I_{1}\right| .
$$

Then $k \geq \delta^{-1}$. Since $\mu \in \mathcal{D}_{K}\left([0,1]^{2}\right)$, we have $\mu\left(I_{1} \times J_{i}\right) \leq K \mu\left(I_{2} \times J_{i}\right)$ for $i \neq k$, and so

$$
\sum_{i \neq k} \mu\left(I_{1} \times J_{i}\right) \leq K \mu\left(I_{2} \times J\right) .
$$


Let $t$ be the index such that

$$
\mu\left(I_{2} \times J_{t}\right)=\min _{1 \leq i \leq k-1} \mu\left(I_{2} \times J_{i}\right) .
$$

Then

$$
\mu\left(I_{2} \times J_{t}\right) \leq \frac{1}{k-1} \mu\left(I_{2} \times J\right)
$$

Fixed $J_{t}$, if necessary, by modifying the previous partition, we may suppose $J_{k}$ is adjacent to $J_{t}$. This modification preserves (8). And we have

$$
\mu\left(I_{1} \times J_{k}\right) \leq K \mu\left(I_{2} \times J_{t}\right) \leq \frac{K}{k-1} \mu\left(I_{2} \times J\right)
$$

It follows from (8) and (9) that

$$
\mu\left(I_{1} \times J\right) \leq \frac{k K}{k-1} \mu\left(I_{2} \times J\right) \leq \frac{K}{1-\delta} \mu\left(I_{2} \times J\right) .
$$

Therefore, we may choose $C(K, \delta)=\frac{K}{1-\delta}$ when $\delta<1 / 2$. The proof of Lemma 8 is complete.

Lemma 9. Let $K \geq 1$ and $\mu \in \mathcal{D}_{K}\left([0,1]^{2}\right)$. Let $\delta>0$. Let $I_{1}, I_{2}$, and $J$ be intervals in $[0,1]$ with $I_{1} \subset I_{2}$ and $\left|I_{2}\right| \leq \delta|J|$. Then

$$
\lambda\left(\frac{\left|I_{1}\right|}{\left|I_{2}\right|}\right)^{\beta} \leq \frac{\mu\left(I_{1} \times J\right)}{\mu\left(I_{2} \times J\right)} \leq 4\left(\frac{\left|I_{1}\right|}{\left|I_{2}\right|}\right)^{\alpha}
$$

where $\lambda, \alpha$, and $\beta$ are constants depending only on $K$ and $\delta$. More precisely, the constant $\alpha$ can be chosen as $\alpha=\log _{2}\left(1+C(K, \delta)^{-1}\right)$.

Proof. Using Lemma 8, the proof is the same as that of Lemma 1 in [19].

Lemma 10. Let $I$ be a closed interval in $\mathbf{R}$. Let $K \geq 1$ and $\mu \in \mathcal{D}_{K}(I)$. Then $\mu$ can be extended to be a $C(K)$-doubling measure on $\mathbf{R}$, where $C(K)$ is a constant depending only on $K$. Moreover, $C(K)$ can be chosen so that $\lim _{K \rightarrow 1} C(K)=1$.

Proof. It is a corollary of Lemma 2.1 of [1].

\section{Proofs of Theorems 2 and 3}

Proof of Theorem 2. Let $E$ and $F$ be subsets of $[0,1]$ with $|E|=|F|=0$. Based on Section 3, the proof goes as follows.

Claim 1. $E \in \mathrm{VT} \Longrightarrow E \times F \in \mathrm{VT}$.

Let $E \in \mathrm{VT}$ and $\mu \in \mathcal{D}\left([0,1]^{2}\right)$. By Lemma 8 , we have $\mu(\cdot \times[0,1]) \in \mathcal{D}([0,1])$. It follows that $\mu(E \times[0,1])=0$, so $\mu(E \times F)=0$. Thus $E \times F \in \mathrm{VT}$.

Claim 2. $E \times F \in \mathrm{VT} \Longrightarrow$ either $E$ or $F \in \mathrm{VT}$.

Suppose neither $E$ nor $F$ is very thin. Then there are $\mu, \nu \in \mathcal{D}([0,1])$ such that $\mu(E) \nu(F)>0$. By Lemma 7 , we have $\mu \times \nu \in \mathcal{D}\left([0,1]^{2}\right)$, for which we have $(\mu \times \nu)(E \times F)=\mu(E) \nu(F)>0$. This shows $E \times F \notin \mathrm{VT}$.

Claim 3. Both $E$ and $F \in \mathrm{MT} \Longrightarrow E \times F \in \mathrm{MT}$.

Since both $E$ and $F \in \mathrm{MT}$, for every $K>1$ there are $\mu, \nu \in \mathcal{D}_{K^{1 / 2}}([0,1])$ such that $\mu(E) \nu(F)>0$. By Lemma 7 , we have $\mu \times \nu \in \mathcal{D}_{K}\left([0,1]^{2}\right)$ with $(\mu \times \nu)(E \times F)=$ $\mu(E) \nu(F)>0$. It follows that $E \times F \in \mathrm{MT}$. 
Claim 4. $E \times F \in \mathrm{MT} \Longrightarrow E \in \mathrm{MT}$.

Given $K>1$, choose a sufficiently small $\delta>0$ such that $(1-\delta) K>1$. Since $E \times F \in \mathrm{MT}$, there is a $(1-\delta) K$-doubling measure $\mu$ on $[0,1]^{2}$ such that $\mu(E \times F)>0$. Let $I$ be an interval in $[0,1]$ of length $|I| \leq \delta$ such that $\mu((E \cap I) \times F)>0$. From the proof of Lemma 8 , the measure $\mu(\cdot \times[0,1])$, defined on $I$, is $K$-doubling on $I$, and for which the set $E \cap I$ has positive measure. Now, using Lemma 10, the above measure on $I$ can be extended to be a $C(K)$-doubling measure $\nu$ on $[0,1]$ such that

$$
\nu(E) \geq \mu((E \cap I) \times[0,1]) \geq \mu((E \cap I) \times F)>0 .
$$

Since $C(K)$ can be chosen so that $\lim _{K \rightarrow 1} C(K)=1$, we get $E \in$ MT.

Proof of Theorem 3. Let $E$ and $F$ be subsets of $[0,1]$ with $|E|>0$ and $|F|>0$. Based on Lemma 7, the proof goes as follows.

Claim 1. $E \times F \in \mathrm{VF} \Longrightarrow E \in \mathrm{VF}$.

If $E \notin \mathrm{VF}$ then there is a $\mu \in \mathcal{D}([0,1])$ such that $\mu(E)=0$. From Lemma 7 we have $\mu \times \mathcal{L} \in \mathcal{D}\left([0,1]^{2}\right)$, where $\mathcal{L}$ denotes the Lebesgue measure on [0,1]. Since $(\mu \times \mathcal{L})(E \times F)=\mu(E) \mathcal{L}(F)=0$, we get $E \times F \notin \mathrm{VF}$, a contradiction.

Claim 2. $E \in \mathrm{MF} \Longrightarrow E \times F \in \mathrm{MF}$.

Since $E \in \mathrm{MF}$, for every $K>1$ there is a $\mu \in \mathcal{D}_{K}([0,1])$ such that $\mu(E)=0$. From Lemma 7 we have $\mu \times \mathcal{L} \in \mathcal{D}_{K}\left([0,1]^{2}\right)$ with $(\mu \times \mathcal{L})(E \times F)=\mu(E) \mathcal{L}(F)=0$. It follows that $E \times F \in \mathrm{MF}$.

\section{Proof of Theorem 4}

Let $E$ be the uniform Cantor set of data $\left\{n_{k}\right\}$ and $\left\{c_{k}\right\}$ and $F$ the uniform Cantor set of data $\left\{m_{k}\right\}$ and $\left\{d_{k}\right\}$, with $|E|>0$ and $|F|>0$. Since Theorem 3 has been proved, it suffices to show the following two claims.

Claim 1. Both $E$ and $F \in \mathrm{VF} \Longrightarrow E \times F \in \mathrm{VF}$.

Let $K \geq 1$ and $\mu \in \mathcal{D}_{K}\left([0,1]^{2}\right)$. Let $C(K, \delta)$ be the constant in Lemma 8. Let $\alpha=\log _{2}\left(1+C(K, 3)^{-1}\right)$. Since both $E$ and $F$ are very fat, by Lemma 4 , we have

$$
\sum_{k=1}^{\infty}\left(n_{k} c_{k}\right)^{\alpha}<\infty \text { and } \sum_{k=1}^{\infty}\left(m_{k} d_{k}\right)^{\alpha}<\infty .
$$

Let $k_{0}$ be an integer such that

$$
\max \left\{\sum_{k=k_{0}}^{\infty}\left(n_{k} c_{k}\right)^{\alpha}, \sum_{k=k_{0}}^{\infty}\left(m_{k} d_{k}\right)^{\alpha}\right\} \leq \frac{1}{16} .
$$

Let $\mathcal{E}_{k}$ be the family of component intervals of level $k$ and $\mathcal{G}_{k}$ be the family of gaps of level $k$ of $E$. Let $\mathcal{F}_{k}$ be the family of component intervals of level $k$ and $\mathcal{V}_{k}$ be the family of gaps of level $k$ of $F$. By (12), for both $E$ and $F$ the length of a gap is smaller than the length of a component interval at the same level $k$, whenever $k \geq k_{0}$.

To show $\mu(E \times F)>0$, it suffices to prove $\mu(S \cap(E \times F))>0$ for some rectangle $S \subset[0,1]^{2}$. Let $I_{k_{0}} \in \mathcal{E}_{k_{0}}$ and $\widetilde{I}_{k_{0}} \in \mathcal{F}_{k_{0}}$. Without loss of generality, suppose $\left|I_{k_{0}}\right| \leq\left|\widetilde{I}_{k_{0}}\right|$. Then we have a unique integer $k_{*} \geq k_{0}$ such that

$$
\left|\widetilde{I}_{k_{*}+1}\right|<\left|I_{k_{0}}\right| \leq\left|\widetilde{I}_{k_{*}}\right|
$$


where $\widetilde{I}_{k_{*}} \in \mathcal{F}_{k_{*}}$ and $\widetilde{I}_{k_{*}+1} \in \mathcal{F}_{k_{*}+1}$. Let $I_{k_{0}}$ and $\widetilde{I}_{k_{*}}$ be fixed as above. The rectangle $S$ is chosen to be

$$
S=I_{k_{0}} \times \widetilde{I}_{k_{*}}
$$

Let $A=\widetilde{I}_{k_{*}} \cap \cup_{k=k_{*}+1}^{\infty} \cup_{J \in \mathcal{V}_{k}} J$ and $B=I_{k_{0}} \cap \cup_{k=k_{0}+1}^{\infty} \cup_{J \in \mathcal{G}_{k}} J$. Then $A$ is the union of gaps of $F$ lying in $\widetilde{I}_{k_{*}}$ and $B$ is the union of gaps of $E$ lying in $I_{k_{0}}$. We easily see that

$$
S \cap(E \times F)=S \backslash\left(\left(I_{k_{0}} \times A\right) \cup\left(B \times \widetilde{I}_{k_{*}}\right)\right) .
$$

Next we estimate $\mu\left(I_{k_{0}} \times A\right)$ and $\mu\left(B \times \widetilde{I}_{k_{*}}\right)$. We first estimate $\mu\left(I_{k_{0}} \times A\right)$. Let $k \geq k_{*}$ be given. Let $I \in \mathcal{F}_{k}$ be a given component interval of $F$ lying in $\widetilde{I}_{k_{*}}$. Let $J_{1}, J_{2}, \cdots, J_{m_{k+1}} \in \mathcal{V}_{k+1}$ be the $m_{k+1}$ gaps lying in $I$. Partition $I$ into $m_{k+1}$ subintervals of equal length and denote them by $J_{1}^{*}, J_{2}^{*}, \cdots, J_{m_{k+1}}^{*}$. Suppose these gaps and intervals have been arranged in ascending order. Then we have for each $1 \leq i \leq m_{k+1}$

$$
J_{i} \subset J_{i}^{*} \text { and } \frac{\left|J_{i}\right|}{\left|J_{i}^{*}\right|}=m_{k+1} d_{k+1} .
$$

Since, as mentioned, the length of a gap of $F$ is smaller than the length of a component interval at the level $k+1$, by (13), one has $\left|J_{i}\right| \leq\left|\widetilde{I}_{k_{*}+1}\right|<\left|I_{k_{0}}\right|$, and so $\left|J_{i}{ }^{*}\right| \leq 3\left|I_{k_{0}}\right|$. It follows from Lemma 9 that

$$
\frac{\mu\left(I_{k_{0}} \times J_{i}\right)}{\mu\left(I_{k_{0}} \times J_{i}^{*}\right)} \leq 4\left(m_{k+1} d_{k+1}\right)^{\alpha}
$$

Summing over all $i$ yields

$$
\frac{\mu\left(I_{k_{0}} \times\left(I \cap \cup_{J \in \mathcal{V}_{k+1}} J\right)\right)}{\mu\left(I_{k_{0}} \times I\right)} \leq 4\left(m_{k+1} d_{k+1}\right)^{\alpha} .
$$

Summing over all $I \in \mathcal{F}_{k}$ lying in $\widetilde{I}_{k_{*}}$ yields

$$
\frac{\mu\left(I_{k_{0}} \times\left(\widetilde{I}_{k_{*}} \cap \cup_{J \in \mathcal{V}_{k+1}} J\right)\right)}{\mu\left(I_{k_{0}} \times\left(\widetilde{I}_{k_{*}} \cap \mathcal{F}_{k}\right)\right)} \leq 4\left(m_{k+1} d_{k+1}\right)^{\alpha},
$$

which gives

$$
\mu\left(I_{k_{0}} \times\left(\widetilde{I}_{k_{*}} \cap \cup_{J \in \mathcal{V}_{k+1}} J\right)\right) \leq 4 \mu(S)\left(m_{k+1} d_{k+1}\right)^{\alpha} .
$$

Now, summing over all $k \geq k_{*}$, we get from (12)

$$
\mu\left(I_{k_{0}} \times A\right) \leq 4 \mu(S) \sum_{k=k_{*}}^{\infty}\left(m_{k+1} d_{k+1}\right)^{\alpha} \leq \frac{\mu(S)}{4} .
$$

Since $\left|I_{k_{0}}\right| \leq\left|\widetilde{I}_{k_{*}}\right|$, by the same argument as that of (15), we have

$$
\mu\left(B \times \widetilde{I}_{k_{*}}\right) \leq \frac{\mu(S)}{4} .
$$

Finally, it follows from (14), (15), and (16) that

$$
\begin{aligned}
\mu(S \cap(E \times F)) & \geq \mu(S)-\mu\left(I_{k_{0}} \times A\right)-\mu\left(B \times \widetilde{I}_{k_{*}}\right) \\
& \geq \mu(S)-\frac{\mu(S)}{4}-\frac{\mu(S)}{4}=\frac{\mu(S)}{2}>0 .
\end{aligned}
$$

Claim 2. $E \times F \in \mathrm{MF} \Longrightarrow$ either $E$ or $F \in \mathrm{MF}$. 
Suppose that neither $E$ nor $F$ is minimally fat. From Lemma 4 there is a constant $\alpha \in(0,1)$ such that

$$
\sum_{k=1}^{\infty}\left(n_{k} c_{k}\right)^{\alpha}<\infty \text { and } \sum_{k=1}^{\infty}\left(m_{k} d_{k}\right)^{\alpha}<\infty
$$

Choose a positive integer $N$ and a constant $K>1$ such that

$$
\alpha \leq \log _{2}\left(1+C(K, 3 / N)^{-1}\right) \quad \text { and } \quad N^{-\log _{2}\left(1+C(K, 3)^{-1}\right)} \log _{2}(N+1) \leq \frac{1}{32} .
$$

Here $C(K, \delta)$ is the constant in Lemma 8 satisfying $\lim _{\delta \rightarrow 0} C(K, \delta)=K$. To show $E \times F \notin \mathrm{MF}$, it suffices to prove $\mu(E \times F)>0$ for all $\mu \in \mathcal{D}_{K}\left([0,1]^{2}\right)$.

Let $\mu \in \mathcal{D}_{K}\left([0,1]^{2}\right)$ be given. Let $k_{0}$ be a positive integer such that

$$
\max \left\{\sum_{k=k_{0}}^{\infty}\left(n_{k} c_{k}\right)^{\alpha}, \sum_{k=k_{0}}^{\infty}\left(m_{k} d_{k}\right)^{\alpha}\right\} \leq \min \left\{\frac{1}{32}, \frac{1}{N}\right\} .
$$

Then for both $E$ and $F$ the length of a gap is smaller than the length of a component interval at the same level $k$, whenever $k \geq k_{0}$. Let $I_{k_{0}} \in \mathcal{E}_{k_{0}}$ and $\widetilde{I}_{k_{0}} \in \mathcal{F}_{k_{0}}$. Without loss of generality, we may suppose $\left|I_{k_{0}}\right| \leq\left|\widetilde{I}_{k_{0}}\right|$. Let an integer $k_{*} \geq k_{0}$, a rectangle $S=I_{k_{0}} \times \widetilde{I}_{k_{*}}, A=\widetilde{I}_{k_{*}} \cap \cup_{k=k_{*}+1}^{\infty} \cup_{J \in \mathcal{V}_{k}} J$, and $B=I_{k_{0}} \cap \cup_{k=k_{0}+1}^{\infty} \cup_{J \in \mathcal{G}_{k}} J$ be defined as those in Claim 1. We are going to to show $\mu(S \cap(E \times F))>0$.

To estimate $\mu\left(I_{k_{0}} \times A\right)$, we divide $A$ into two parts by

$$
A=\left(\widetilde{I}_{k_{*}} \cap \bigcup_{k=k_{* *}+1}^{\infty} \bigcup_{J \in \mathcal{V}_{k}} J\right) \cup\left(\widetilde{I}_{k_{*}} \cap \bigcup_{k=k_{*}+1}^{k_{* *}} \bigcup_{J \in \mathcal{V}_{k}} J\right):=A_{1} \cup A_{2},
$$

where $k_{* *}>k_{*}$ is the biggest integer such that $k_{* *}-k_{*} \leq \log _{2}(N+1)$. This choice of $k_{* *}$ ensures that

$$
N\left|\widetilde{I}_{k_{* *}}\right| \leq\left|\widetilde{I}_{k_{*}+1}\right|<\left|I_{k_{0}}\right|
$$

for every $\widetilde{I}_{k_{* *}} \in \mathcal{F}_{k_{* *}}$. Now, using Lemma 9 with $\delta=3 / N$, by the same argument as that of (15) we get from (18) and (19) that

$$
\mu\left(I_{k_{0}} \times A_{1}\right) \leq 4 \mu(S) \sum_{k=k_{* *}+1}^{\infty}\left(m_{k} d_{k}\right)^{\log _{2}\left(1+C(K, 3 / N)^{-1}\right)} \leq \frac{\mu(S)}{8} .
$$

As for $\mu\left(I_{k_{0}} \times A_{2}\right)$, by using Lemma 9 with $\delta=3$ and by the same argument as that of (15) we get from (18) and (19) that

$$
\begin{aligned}
\mu\left(I_{k_{0}} \times A_{2}\right) & \leq 4 \mu(S) \sum_{k=k_{*}+1}^{k_{* *}}\left(m_{k} d_{k}\right)^{\log _{2}\left(1+C(K, 3)^{-1}\right)} \\
& \leq 4 \mu(S) N^{-\log _{2}\left(1+C(K, 3)^{-1}\right)} \log _{2}(N+1) \leq \frac{\mu(S)}{8} .
\end{aligned}
$$

Therefore,

Similarly, we have

$$
\mu\left(I_{k_{0}} \times A\right) \leq \frac{\mu(S)}{4}
$$

$$
\mu\left(B \times I_{k_{*}}\right) \leq \frac{\mu(S)}{4}
$$


It follows that

$$
\mu(S \cap(E \times F)) \geq \mu(S)-\mu\left(I_{k_{0}} \times A\right)-\mu\left(B \times \widetilde{I}_{k_{*}}\right) \geq \frac{\mu(S)}{2}>0 .
$$

This completes the proof.

\section{References}

[1] Buckley, S. M., B. Hanson, and P. MacManus: Doubling for general sets. - Math. Scand. 88, 2001, 229-245.

[2] Csörnyei, M., and V. Suomala: On Cantor sets and doubling measures. - J. Math. Anal. Appl. (to appear).

[3] Falconer, K. J.: Techniques in fractal geometry. - John Wiley \& Sons, 1997.

[4] Garnett, J., R. Killip, and R. Schul: A doubling measure on $\mathbf{R}^{d}$ can charge a rectifiable curve. - Proc. Amer. Math. Soc. 138, 2010, 1673-1679.

[5] Hakobyan, H. A.: Cantor sets minimal for quasisymmetric maps. - J. Contemp. Math. Anal. $41: 2,2006,5-13$.

[6] HAn, D., L. WANG, and S. Wen: Thickness and thinness of uniform Cantor sets for doubling measures. - Nonlinearity 22, 2009, 545-551.

[7] Heinonen, J.: Lectures on analysis on metric spaces. - Springer-Verlag, New York, 2001.

[8] Hu, M., and S. Wen: Quasisymmetrically minimal uniform Cantor sets. - Topology Appl. $155,2008,515-521$.

[9] Luukkainen, J., and E. Saksman: Every complete doubling space carries a doubling measure. - Proc. Amer. Math. Soc. 126, 1998, 531-534.

[10] Kaufman, R., J.-M. Wu: Two problems on doubling measures. - Rev. Math. Iberoam. 11, $1995,527-545$.

[11] Käenmäki, A., T. Rajala, and V. Suomala: Existence of doubling measures via generalized nested cubes. - Proc. Amer. Math. Soc. 140, 2012, 3275-3281.

[12] Ojala, T., T. Rajala, and V. Suomala: Thin and fat sets for doubling measures in metric spaces. - Studia Math. 208, 2012, 195-211.

[13] Peng, F. J., and S. Y. Wen: Fatness and thinness of uniform Cantor sets for doubling measures. - Sci. China Math. 54, 2011, 75-81.

[14] Staples, S., and L. Ward: Quasisymmetrically thick sets. - Ann. Acad. Sci. Fenn. Math. 23, 1998, 151-168.

[15] TukiA, P.: Hausdorff dimension and quasisymmetrical mappings. - Math. Scand. 65, 1989, $152-160$.

[16] VÄISÄLÄ, J.: Lectures on $n$-dimensional quasiconformal mappings. - Lecture Notes in Math. 229, Springer-Verlag, Berlin-Heidelberg-New York, 1971.

[17] Vol'Berg, A. L., and S. V. Konyagin: On measures with the doubling condition. - Math. USSR-Izv. 30, 1988, 629-638.

[18] WANG, W., S. Wen, and Z. WEN: Note on atomic doubling measures, quasisymmetrically thin sets and thick sets. - J. Math. Anal. Appl. 385, 2012, 1027-1032.

[19] Wu, J.-M.: Null sets for doubling and dyadic doubling measures. - Ann. Acad. Sci. Fenn. Math. 18, 1993, 77-91.

[20] Wu, J.-M.: Hausdorff dimension and doubling measures on metric spaces. - Proc. Amer. Math. Soc. 126, 1998, 1453-1459.

Received 23 May 2012 • Accepted 12 November 2012 